hautnah $2013 \cdot 12: 2$

DOI 10.1007/s12326-013-0072-2

() Springer-Verlag 2013
I. Schlager

Redaktion hautnah

\title{
Prävention ist nicht nur Privatsache!
}

Vorsorgen statt reparieren - so soll die Zukunft der Gesundheitsversorgung der heimischen Bevölkerung aussehen. Egal ob Politiker oder andere Entscheidungsträger im Gesundheitswesen - alle betonen seit Jahren, wie wichtig ihnen das Thema Prävention ist, wie viele Kosten dadurch eingespart werden können und wie sehr die Gesellschaft davon profitieren würden.

In Zahlen lässt sich dieses allgemeine Interesse allerdings schwer nachvollziehen: Laut Statistik Austria beliefen sich 2011 die gesamten heimischen Gesundheitsausgaben auf mehr als 32 Milliarden Euro. Davon flossen nicht einmal 1,5 Prozent in den Bereich „Prävention“, und vielmehr wird es offenbar auch in Zukunft nicht werden. Damit zählt Österreich in Europa zu den Schlusslichtern.

\section{Schlechte Rahmenbedingungen}

Und das ist nur die eine Seite der Medaille: Wirkliche Gesundheitsvorsorge bedeutet viel mehr als rein medizinische Maßnahmen wie Impfprogramme und Vorsorgeuntersuchungen; auch mehr als ständige Appelle an die „Eigenverantwortung“ der Bürger. Primärprävention setzt ganz am Anfang an und umfasst grundlegende gesellschaftspolitische Maßnahmen, um die Gesundheit zu erhalten und zu fördern: Bildung, Aufklärung, Erziehung, Information. Und da schaut's düster aus.
Beispiel Bildung/Erziehung: Ob PISA, PIRLS oder TIMSS - internationale Bildungsstudien offenbaren seit Jahren gravierende Schwächen der österreichischen Schüler, ohne dass dies sichtbare Konsequenzen hätte. (Frage: Geht es beim Thema „Bildungsreform“ eigentlich irgendwann einmal um etwas Anderes als die Bedürfnisse der Lehrer?) Auch Themen wie mehr wöchentliche Turnstunden, gesundes Schulbuffet, zusätzliches Sportangebot oder Nahrungsmittelkunde als Unterrichtsfach werden halbherzig angegangen. Die meisten entsprechenden Projekte basieren auf der Eigeninitiative von Schulleitern und Lehrern.

Beispiel Information/Aufklärung: Wenn ich mir einen neuen Wecker kaufe, werde ich in der Gebrauchsanweisung eindringlich davor gewarnt, das Gerät anzuzünden, zu schlucken oder in der Mikrowelle zu garen. Auf der anderen Seite ist eine für den Durchschnittsbürger einigermaßen verständliche Lebensmittelkennzeichnung offenbar nicht durchsetzbar, ein Ampelsystem wie in Großbritannien illusorisch.

\section{Lieber reich und gesund als arm und krank}

Personaleinsparungen, All-inclusiveArbeitsverträge, „leistungsgerechte“ Entlohnung und flexible Arbeitszeiten bei gleichzeitigen Reallohnsenkungen - der Druck auf die Arbeitnehmer ist in den letzten Jahren massiv gestiegen. Die Folgen sind unter anderem eine wachsenden Kluft zwischen arm und reich und eine steigende Anzahl an „Working Poor“ - Menschen, deren Gehalt nicht mehr ausreicht, um ihren Lebensunterhalt zu bestreiten. Dieser Entwicklung (glaubhaft!) entgegenzutreten, wäre eine echte Aufgabe für die kommende Regierung.

Klassenkampfrhetorik? Vielleicht, aber alle relevanten Studien zeigen einen direkten Zusammenhang von Wohlstand und Gesundheit. Und bei allem Respekt für die immensen medizinischen Fortschritte: Zum Anstieg der Lebenserwartung in den vergangenen 150 Jahren haben nicht zuletzt der Aufbau der Sozialsysteme, der Abbau von sozialen Ungleichheiten, die Absicherung der Menschen vor den Folgen von Krankheit, der Ausbau der Bildungssysteme und der wachsende Wohlstand beigetragen,

meint Ihr

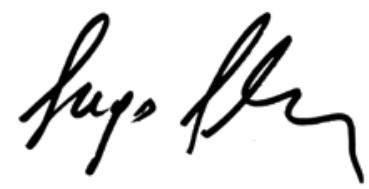

Ingo Schlager

Redaktion hautnah

ingo.schlager@chello.at 